\title{
Associations between mandibular torus and types of temporomandibular disorders, and the clinical usefulness of temporary splint for checking bruxism
}

\author{
Hee-Min Lee ${ }^{1}$, Dong-Woo Kang ${ }^{1}$, Pil-Young Yun ${ }^{1}$, Il-hyung Kim ${ }^{1,2^{*}}$ and Young-Kyun Kim ${ }^{1,3^{*}}$
}

\begin{abstract}
Background: Occlusal stress from oral parafunctional habits is one of the causes of temporomandibular disorders (TMD) and mandibular torus (MT). Although some studies have investigated the correlation between TMD and MT, understanding of the relationships between types of TMD and MT is insufficient. Therefore, we conducted this study to investigate the associations between presence of MT and TMD types.
\end{abstract}

Methods: This study included 77 patients diagnosed with TMD who first visited our clinic for TMD between March 2019 and July 2020. Among them, 30 (38.9\%) had MT, and 54 (70.1\%) had oral parafunction. Parafunctional activity during sleep was confirmed using a temporary splint for checking bruxism (TSCB).

Results: The relationship between prevalence of MT and oral parafunction in TMD patients was not statistically significant $(P=0.131)$, but the odds ratio was relatively high at 2.267. An analysis of TMD type revealed that Type I, which is classified as myalgia of the masticatory muscles, and MT had a significant association $(P=0.011)$. We fabricated a TSCB for 27 patients to wear during sleep and confirmed that 23 (85.2\%) had nocturnal bruxism. The TSCB results and presence of MT showed a significant relationship $(P=0.047)$.

Conclusion: Through the results of this study, clinicians may consider the hyperactivity of masticatory muscles in the presence of MT when treating TMD patients. In addition, TSCB has a great diagnostic value as it can be easily manufactured and be useful for discovering pre-existing oral parafunctions that patients are not aware of.

Keywords: Mandibular torus, Temporomandibular disorders, Oral parafunctional habits, Bruxism, Clenching, Splint, Temporary splint for checking bruxism

\section{Background}

Temporomandibular disorders (TMD) are a group of disorders of the masticatory muscles of the temporomandibular region, joints, and related anatomical structures. TMD results from macrotrauma and microtrauma;

\footnotetext{
*Correspondence: ddsmaxman@gmail.com; kyk0505@daum.net ${ }^{1}$ Department of Oral and Maxillofacial Surgery, Section of Dentistry, Seoul National University Bundang Hospital, 82 Gumi-ro 173beon-gil, Bundang-gu, Seongnam 13620, Korea

Full list of author information is available at the end of the article
}

the latter occurs when a load is repeatedly applied to a joint's structure over a long period of time, resulting in destruction due to hypoxia from insufficient blood flow [1]. Masticatory muscle pain caused by excessive use also is considered a cause of TMD [2]. Swelling occurs as a result of increased intramuscular pressure and continuous activation of several motor units to produce a lowered threshold for excessive load. When these muscles are damaged, symptoms of inflammation such as pain, swelling, and tenderness occur in the 
temporomandibular region [3]. Therefore, among various causes, microtrauma continuously applied to the region due to excessive masticatory function and occlusal stress due to oral parafunction is a major risk factor for TMD $[4,5]$.

Mandibular torus (MT) is a benign bone growth with clear border and slow growing rate. It mostly appears on lingual side of bilateral mandible premolar. Although the cause of MT is not understood, occlusal stress and excessive masticatory function have been suggested as the major factors [6-8]. Therefore, it is convincing that oral parafunctions such as clenching or teeth grinding are closely related to the occurrence of MT as well as TMD. Some studies have reported the relationship among TMD, oral parafunctions, and MT $[6,9]$, while some studies have reported on the relationship between oral parafunctions and MT $[7,9,10]$.

Nevertheless, studies have not focused on the relationship between subtype of TMD and of MT. Therefore, in this study, we classified TMD patients by subtype and analyzed the concordant presence of MT. Through this study, we would like to highlight the MT's diagnostic value hidden in, which is often overlooked in clinical practice. Also, we would like to introduce a simple and useful device called Temporary Splint for Checking Bruxism (TSCB) that we use to diagnose TMD patients.

\section{Methods}

\section{Study subjects}

The subjects were patients diagnosed with TMD who first visited the Oral and Maxillofacial Surgery Department of Seoul National University Bundang Hospital between March 2019 and July 2020. The criteria for excluding research subjects were as follows: (1) history of maxillofacial tumor, (2) history of facial trauma, (3) absence of one or more molars that had not been restored, and (4) history of botulinum toxin injection within the last six months. The study was approved by the Seoul National University Bundang Hospital Institutional Review Board (IRB) (B-2010-642-104) and was performed in accordance with the Helsinki guidelines. Informed consent was obtained from all subjects.

\section{Research category}

\section{Temporomandibular disorders}

All the subjects were diagnosed with TMD by one calibrated and criterion standardized oral and maxillofacial surgeon who performed the same clinical exam for each. The initial visit consisted of a questionnaire on their pain level and duration as well as clinical examination on TMD-related symptoms using Diagnostic Criteria for Temporomandibular Disorders (DC/TMD). Then, orthopantomography and temporomandibular panoramic radiography were taken for radiological examination and cone beam computed tomography (CBCT) and bone scan or single photon emission computed tomography (SPECT) were used as needed. After every inspection, patients were diagnosed as one or more of TMD type I to IV, a detailed diagnostic criteria based on the TMD classification system of the Japanese Society for Temporomandibular Joint (JSTMJ, 2013). According to the system, TMD is classified into myalgia of the masticatory muscle (Type I), arthralgia (Type II), disc derangement (Type III), and osteoarthrosis or osteoarthritis (Type IV) (Table 1). Each type in this taxonomy corresponds to a classification of DC/TMD, and Type I is masticatory muscle disorder, and Type II, III, IV are joint pain, joint disorders, and joint diseases respectively in temporomandibular joint disorders.

\section{Mandibular torus}

Five dentists who had completed the training for this investigation performed oral examination during the first visit to determine the presence of a MT. Patients were classified to have MT only when bilateral nodular mass above the mylohyoid ridge one the lingual side of the mandible in the canine to premolar area was clearly distinguishable by naked eye.

\section{Oral parafunctional habits}

Patients were considered to have oral parafunctions if any one of the following condition was present: (1) Clenching and/or bruxing, day or night, either self-reported or reported by their sleeping partners, (2) Patients with positive results of Temporary Splint for Checking Bruxism (TSCB) with relevant symptoms and signs through questionnaire and clinical examination at the first visit.

\section{Temporary splint for checking bruxism}

No matter how much patients were aware of their own damaging oral habits, TSBC was fabricated and delivered to be worn during night time if they reported to

Table 1 Classification of temporomandibular joint disorders (2013, JSTMJ*)

\begin{tabular}{ll}
\hline Type & Contents \\
\hline I & Myalgia of a masticatory muscle \\
III & Arthralgia of the temporomandibular joint \\
& Temporomandibular joint disc derangement \\
& a. with reduction \\
IV & O. without reduction \\
& Osteoarthrosis/osteoarthritis of the tempo- \\
& romandibular joint \\
\hline
\end{tabular}

*Japanese Society for Temporomandibular Joint 
have, pain or tenderness during palpation in the regions of masticatory muscle when they open their mouth immediately after waking up. In addition, if intraoral exam showed scalloped tongue, linea alba on the buccal mucosa, and multiple occlusal attrition, abfractions, and hypersensitivity teeth that cannot be explained as others, TSCB was prescribed assuming that the patient had oral parafunctions.

TSCB is a transparent and hard Omnivac splint with a thickness of $0.6 \mathrm{~mm}$. Alginate impressions were taken at the first visit for the patients in need, and immediately fabricated and given to patients. After wearing it while sleeping for one week, the number of scratched or perforated points observed on the surface of the device at the next visit was applied as the result of TSCB (Fig. 1). Cases with more than one TSCB result were regarded to have oral parafunctions.

\section{Statistical analysis}

For statistical analysis, IBM SPSS version 25.0 (SPSS Inc., Chicago, IL, USA) was used, and the statistical significance level was set to $95 \%$. Statistical analysis was
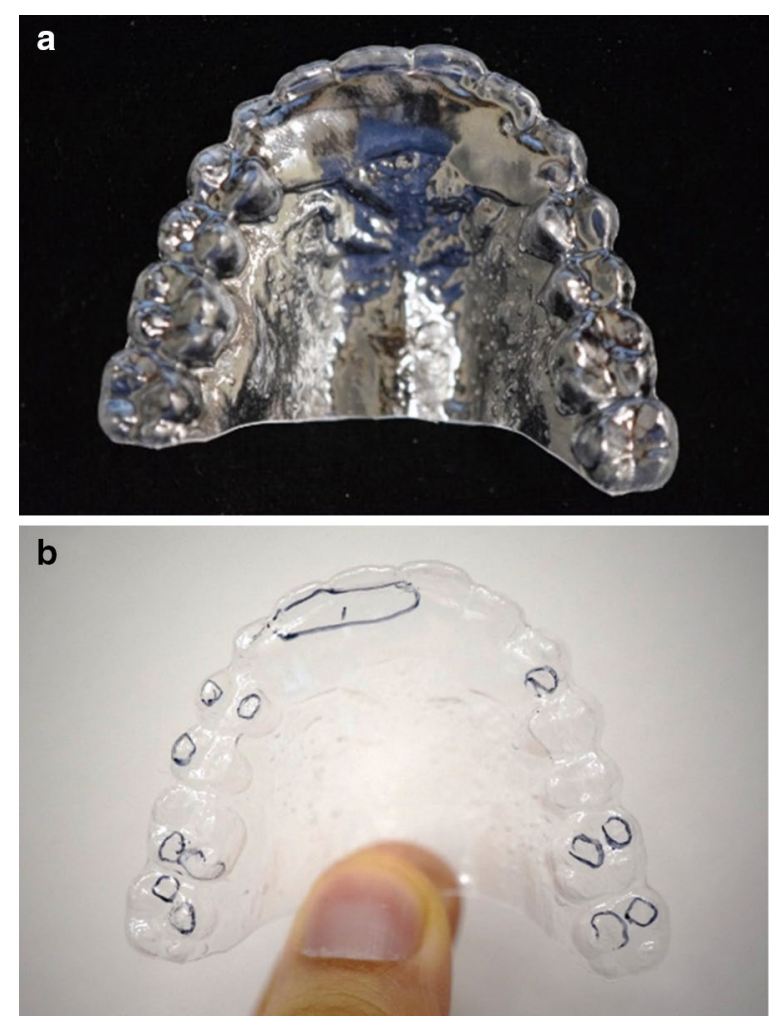

Fig. 1 Temporary Splint for Checking Bruxism (TSCB) used to detect nocturnal oral parafunctions that patients are unaware of $\mathbf{a}$ The TSCB is a hard Omnivac splint with a 0.6-mm thickness. $\mathbf{b}$ After using the device during sleep for a week, several marks are identified on the surface, indicating that the patient is a bruxer at night performed on sex, age, duration of TMD-related symptoms, TMD type, oral parafunction, and TSBC results for the presence or absence of MT. Logistic regression analysis was used for analysis of sex, age, and TMD type, and a chi-square test was carried out to analyze oral parafunction. For analysis of duration of TMD-related symptoms and TSBC results, a linear-by-linear association was used.

\section{Results}

The study comprised 77 patients, all Koreans: 27 males (35.1\%) and 50 females (64.9\%) with an average age of 41.7 (13-84) years. Of the total patients, $30(38.9 \%)$ had MT, and 54 (70.1\%) demonstrated oral parafunction. The average age of patients with MT was 37.7 (13-67) years. In addition, 11 of 27 males (40.7\%) and 19 of 50 females (38.0\%) had MT. Oral parafunction was confirmed in 20 of 27 males (74.1\%) and in 38 of 50 females (76.0\%). Age and sex were not significantly associated with MT. In addition, there was no statistical significance between MT and oral parafunction, but the odds ratio was relatively high at 2.267 (Table 2 ).

In the subjects, there were 10 distinct combinations of TMD types, with Types I and III being the most common (Table 3). When classifying the participants by TMD type, Type I TMD was the most common in 51 (49.0\%) patients, followed by Type III in 44 (38.6\%), Type IV in 33 (39.4\%), and Type II in 8 (37.5\%) participants. Statistical analysis confirmed that MT was significantly associated with TMD Type I $(P=0.011)$ (Table 4$)$.

Interviews and clinical examinations of 77 TMD patients confirmed that 31 patients had parafunction. Of the remaining 46 patients, 27 were suspected to have oral parafunction and received a TSCB. After one week, 23 patients $(85.2 \%)$ showed positive results on TSCB, and the score was $3.07 \pm 2.67$ out of 0 to 10 range. Overall, 54 patients were found to have oral parafunctional habits. It was confirmed that the increase of the TSCB result was statistically significant with the occurrence of MT $(P=0.047)$ (Table 5).

Table 2 Demographics, mandibular torus and oral parafunctions in patients with TMD

\begin{tabular}{lllll}
\hline & Male & Female & Total & P-value \\
\hline Age & 43.8 & 40.7 & 41.76 & 0.108 \\
Gender & $27(35.1 \%)$ & $50(64.9 \%)$ & $77(100 \%)$ & 0.817 \\
Parafunctions & $17 / 27(34.5 \%)$ & $37 / 50(76.0 \%)$ & $54 / 77(70.1 \%)$ & 0.131 \\
$\begin{array}{l}\text { Mandibular } \\
\text { torus* }\end{array}$ & $11 / 27(40.7 \%)$ & $19 / 50(38.0 \%)$ & $30 / 77(38.9 \%)$ & - \\
\hline
\end{tabular}

*Age, gender, and parafunctions were not statistically significant with mandibular torus, respectively $(P>0.05)$ 
Table 3 Combinations by TMD types in the subject group

\begin{tabular}{lllllllllllll}
\hline & I, III & I & I, III, IV & IV & III & I, IV & III, IV & I, II & II, III & I, II, III & Total \\
\hline No. of patients & 17 & 11 & 10 & 10 & 8 & 7 & 6 & 5 & 2 & 1 & 77 \\
\hline
\end{tabular}

Table 4 Presence of mandibular torus according to each type of TMD

\begin{tabular}{lccc}
\hline TMD type & Count & Presence of torus & $P$-value \\
\hline I $^{*}$ & 51 & $25(49.0 \%)$ & 0.011 \\
II & 8 & $3(37.5 \%)$ & 0.992 \\
III & 44 & $17(38.6 \%)$ & 0.856 \\
IV & 33 & $13(39.4 \%)$ & 0.428 \\
\hline
\end{tabular}

${ }^{*}$ Statistically significant difference with mandibular torus $(P<0.05)$

The duration of TMD-related symptoms investigated from the subjects was classified into less than 6 months, 6 months to 1 year, 1 to 2 years, 2 to 3 years, and 3 years or longer. Among them, 3 years or more was the most common, followed by less than 6 months, 1 to 2 years, 6 months to 1 year, and 2 to 3 years. No statistically significant value was observed for the correlation between the presence of MT and the duration of TMD-related symptoms $(P=0.940)$ (Table 6).

\section{Discussion}

Several studies have reported a prevalence of bony exostosis in the oral cavity, including mandibular and palatal torus, and buccal exostosis, between 12 and 33\% [11-13]. In particular, there is a higher incidence of MT among East Asians, Eskimoans, Mongolians, and Japanese; white people have a higher incidence than black people, demonstrating a large racial difference [14-17].
The most widely accepted theory of MT development is multi-factorial, including genetic and environmental factors. Eggen et al. reported MT to be affected by about $30 \%$ genetic factors and $70 \%$ environmental factors [18]. Other researchers have implicated factors such as trauma, drugs, infections, occlusal stress, and nutrition in occurrence of MT [19-21]. Among these, occlusal stress and excessive bite force have been presented as the main environmental factors involved in MT $[6,18]$.

Clifford et al. reported a close correlation between MT and parafunctional habits, and Sirirungrojying et al. suggested that MT could be used as a marker of TMD $[6,22]$. In this study, the prevalence of MT $(38.9 \%)$ in patients with TMD was higher than that (1.1 to 9.4\%) in the general population [10, 15, 23, 24]. In addition, the predicted prevalence of sleep bruxism in general adults was 5.5 to $15.3 \%$ [25, 26], while the proportion of TSCB-proven sleep bruxism in TMD patients in this study was $29.9 \%$. That is to say, TMD patients in this study showed higher prevalence of MT and nocturnal bruxism compared to the general population. This can be interpreted as a result of excessive and sustained occlusal force seen in TMD patients. We found no significant relationship between oral parafunctional habits and presence of MT in TMD patients, but the odds ratio had a high value of 2.267 , which indicates a similar trend to the results of previous studies $[6,9,22]$.

Table 5 Presence of mandibular torus according to the results of temporary splint for checking bruxism (TSCB) in patients with TMD

\begin{tabular}{|c|c|c|c|c|c|c|c|c|c|c|}
\hline TSCB results ${ }^{*}$ & 0 & 1 & 2 & 3 & 4 & 5 & 6 & 7 & 8 & 10 \\
\hline No. of patients & 4 & 7 & 3 & 2 & 4 & 2 & 2 & 1 & 1 & 1 \\
\hline No. of patients with torus & $1(25 \%)$ & $2(28.5 \%)$ & $0(0 \%)$ & $0(0 \%)$ & $2(50 \%)$ & $2(100 \%)$ & $2(100 \%)$ & $1(100 \%)$ & $1(100 \%)$ & $\begin{array}{l}0 \\
10 \%\end{array}$ \\
\hline
\end{tabular}

*Statistically significant association with mandibular torus $(P<0.05)$

Table 6 Duration of TMD-related symptoms and presence of torus mandibularis in patient with TMD

\begin{tabular}{|c|c|c|c|c|c|c|}
\hline \multirow[t]{2}{*}{ Mandibular torus } & \multicolumn{6}{|c|}{ Duration of symptoms ${ }^{*}$} \\
\hline & $<6$ months & 6 months to 1 year & 1 to 2 years & 2 to 3 years & $>3$ years & Sum \\
\hline Present & 6 & 4 & 5 & 4 & 11 & 30 \\
\hline None & 11 & 4 & 9 & 3 & 20 & 47 \\
\hline Sum & 17 & 8 & 14 & 7 & 31 & 77 \\
\hline
\end{tabular}

*No statistically significant difference with mandibular torus $(P<0.05)$ 
This study is the first to classify TMD patients by subtype and to investigate their relationship with MT. The taxonomic classification for TMD of JSTMJ used in this study, although it is unfamiliar in the Western world, is thought to be meaningful in that it helps TMD be understood by dividing it into four major categories. We confirmed that TMD Type I, which involves myalgia of the masticatory muscles, had a significant association with MT. Based on Wolff's Law, which holds that the bone undergoes remodeling as load increases and becomes stronger over time [27], this result can be explained. Singh et al. also argued that MT is developed by overworking of TMJ, leading to prolonged overload and activates osteoblasts to deposit bone, which seems to be a theory applicable to our results [28]. No meaningful association was found between duration of TMD symptoms and presence of MT in this study. As previously mentioned, MT occurs due to a persistent increased occlusal load, so it was expected that MT would be more frequent as TMD symptom retention period increased; however, our findings did not support this. Therefore, it is better to say that TMD is a complex disorder that should be understood by its subtype.

In this study, retention of MT according to sex in TMD patients was not significant. However, previous studies have shown that MT is diagnosed more often in women $[12,15,17,23,24,29]$. This is thought to be due to the involvement of other factors contributing to the retention of MT since this study was conducted in patients diagnosed with TMD.

It has been negatively recognized whether electromyography (EMG) can be usefully used in diagnosing TMD [30, 31]. However, recent literatures have suggested that EMG values of masticatory muscle have reliable diagnostic sensitivity in pain-related TMD patients [32, 33]. Although EMG was not used in this study, it would be better if EMG be used in the course of TMD treatment in the future by referring to these studies.

TSCB can both reveal and quantify the degree of unconscious oral parafunctions that occur at night. Since we were able to detect previously unnoticed oral parafunctions during sleep in 23 people $(85.1 \%)$ by prescribing a TSCB to 27 patients, we suggest that this device be used as a simple but useful diagnostic tool. Our results indicate that the prevalence of MT significantly increases as TSCB results increase. Accordingly, the more severe is the abnormal oral function, the more common is development of MT. In addition, we found that patients who had used a TSCB had a very high rate of consent to continued stabilization splint treatment despite the high cost. Therefore, TSCB is a convenient and useful tool that can be used to diagnose and treat TMD.
MT and oral parafunctional habits were interrelated in TMD patients and were prominent in TMD Type I presenting masticatory muscle problems and these results can be useful when treating TMD patients. However, patients with MT are often unaware of its existence because of lack of symptoms or discomfort. Clinically, MT has not been considered important except in special situations, such as before the manufacture of dentures. Clinicians need to suspect that TMD patients with MT have parafunction and excessive activity in the masticatory muscle. This study is expected to increase understanding of MT and promote related research in the future.

\section{Conclusion}

We confirmed a close relationship between TMD Type I and presence of MT. Moreover, we suggest that TSCB should be considered as a useful diagnostic device that can be easily manufactured and applied to provide more comprehensive diagnosis to TMD patients.

\section{Acknowledgements \\ Assistance provided by Julie Olivia Chang at Columbia University College of Dental Medicine for this project was greatly appreciated.}

\section{Authors' contributions}

DWK, IHK, and YKK designed the study. YKK was the calibrated examiner of DC/TMD and extensively trained the remaining four. HML and DWK collected and inputted study data. IHK and PYY performed all analyses and contributed to the data analysis strategy. HML wrote the manuscript, while IHK and YKK contributed to the interpretation of results and write-up of the manuscript. $I H K$ and YKK revised the manuscript. All authors read and approved the final manuscript.

\section{Funding}

None.

\section{Availability of data and materials}

The datasets used and/or analyzed in this study are available from the corresponding author upon reasonable request.

\section{Declarations}

\section{Ethics approval and consent to participate}

The study was performed in accordance with the Helsinki guidelines. All participants provided written informed consent for participation in this research, which was approved by the Institutional Review Board at Seoul National University Bundang Hospital. All authors read and approved the final manuscript.

\section{Consent for publication}

Not applicable.

\section{Competing interests}

The authors declare that they have no competing interests.

\section{Author details}

${ }^{1}$ Department of Oral and Maxillofacial Surgery, Section of Dentistry, Seoul National University Bundang Hospital, 82 Gumi-ro 173beon-gil, Bundang-gu, Seongnam 13620, Korea. ${ }^{2}$ Office of Human Resources Development, Armed Forces Capital Hospital, Armed Forces Medical Command, Seongnam, Korea. ${ }^{3}$ School of Dentistry and Dental Research Institute, Seoul National University, Seoul, Korea. 
Received: 12 January 2021 Accepted: 5 April 2021

Published online: 09 April 2021

\section{References}

1. Yun PY, Kim YK. The role of facial trauma as a possible etiologic factor in temporomandibular joint disorder. J Oral Maxillofac Surg. 2005:63:1576-83.

2. Okeson JP, de Leeuw R. Differential diagnosis of temporomandibular disorders and other orofacial pain disorders. Dent Clin North Am. 2011;55:105-20.

3. Agha-Hosseini F, Sheykhbahaei N, Mirzaii-Dizgah I, Fatehi F. The efficacy of oral habit modification on headache. J Korean Assoc Oral Maxillofac Surg. 2017:43:401-6.

4. Ommerborn MA, Giraki M, Schneider C, Fuck LM, Zimmer S, Franz M, Raab WH, Schaefer R. Clinical significance of sleep bruxism on several occlusal and functional parameters. Cranio. 2010;28:238-48.

5. Zhang ZK, Ma XC, Gao S, Gu ZY, Fu KY. Studies on contributing factors in temporomandibular disorders. Chin J Dent Res. 1999:2:7-20.

6. Sirirungrojying S, Kerdpon D. Relationship between oral tori and temporomandibular disorders. Int Dent J. 1999:49:101-4.

7. De Luca CG, de Freitas ST, Schuldt Filho G, Vieira RS. Association between mandibular torus and parafunctional activity. Int I Stomatol Occlusion Med. 2013;6:43-9.

8. Khan S, Shah SAH, Ali F, Rasheed D. Concurrence of Torus Palatinus, Torus Mandibularis and Buccal Exostosis. J Coll Physicians Surg Pak. 2016;26:111-3.

9. Jeong CW. Oral tori in patients with temporomandibular disorders and parafunctional habits. J Oral Maxillofac Surg. 2014;72:e222-3.

10. Prasad RG, Sharma N, Prakash R. Prevalence of Torus mandibularis and its association with para-functional activity in tertiary care centre in Shimla, HP, India: a hospital based cross sectional study. J Dent Res Rev. 2020;7:177.

11. Morrison MD, Tamimi F. Oral tori are associated with local mechanical and systemic factors: a case-control study. J Oral Maxillofac Surg 2013;71:14-22

12. AlZarea BK. Prevalence and pattern of torus palatinus and torus mandibularis among edentulous patients of Saudi Arabia. Clin Interv Aging. 2016;11:209-13

13. Kumar Singh A, Sulugodu Ramachandra S, Arora S, Dicksit DD, Kalyan CG, Singh P. Prevalence of oral tori and exostosis in Malaysian population-a cross-sectional study. J Oral Biol Craniofac Res. 2017;7:158-60.

14. Jarvis A, Gorlin RJ. Minor orofacial abnormalities in an Eskimo population. Oral Surg Oral Med Oral Pathol. 1972;33:417-27.

15. Reichart PA, Neuhaus F, Sookasem M. Prevalence of torus palatinus and torus mandibularis in Germans and Thai. Community Dent Oral Epidemiol. 1988;16:61-4.

16. Jainkittivong A, Apinhasmit W, Swasdison S. Prevalence and clinical characteristics of oral tori in 1,520 Chulalongkorn University Dental School patients. Surg Radiol Anat. 2007;29:125-31.
17. Yoshinaka M, Ikebe K, Furuya-Yoshinaka M, Maeda Y. Prevalence of torus mandibularis among a group of elderly Japanese and its relationship with occlusal force. Gerodontology. 2014;31:117-22.

18. Eggen S. Torus mandibularis: an estimation of the degree of genetic determination. Acta Odontol Scand. 1989:47:409-15.

19. Eggen S, Natvig B. Relationship between torus mandibularis and number of present teeth. Scand J Dent Res. 1986:94:233-40.

20. Eggen S, Natvig B. Concurrence of torus mandibularis and torus palatinus. Scand J Dent Res. 1994:102:60-3.

21. Jainkittivong A, Langlais RP. Buccal and palatal exostoses: prevalence and concurrence with tori. Oral Surg Oral Med Oral Pathol Oral Radiol Endod. 2000:90:48-53.

22. Clifford T, Lamey PJ, Fartash L. Mandibular tori, migraine and temporomandibular disorders. Br Dent J. 1996:180:382-4.

23. Hiremath VK, Husein A, Mishra N. Prevalence of torus palatinus and torus mandibularis among Malay population. J Int Soc Prev Community Dent. 2011;1:60-4.

24. Patil S, Maheshwari S, Khandelwal SK. Prevalence of torus palatinus and torus mandibularis in an Indian population. Saudi J Oral Sci. 2014;1:94.

25. Maluly M, Andersen ML, Dal-Fabbro C, Garbuio S, Bittencourt L, de Siqueira JT, Tufik S. Polysomnographic study of the prevalence of sleep bruxism in a population sample. J Dent Res. 2013;92:97S-103S.

26. Manfredini D, Winocur E, Guarda-Nardini L, Paesani D, Lobbezoo F. Epidemiology of bruxism in adults: a systematic review of the literature. J Orofac Pain. 2013;27:99-110.

27. Frost HM. Wolff's Law and bone's structural adaptations to mechanical usage: an overview for clinicians. Angle Orthod. 1994;64:175-88.

28. Singh GD. On the etiology and significance of palatal and mandibular tori. Cranio. 2010;28:213-5.

29. Bernaba JM. Morphology and incidence of torus palantinus and mandibularis in Brazilian Indians. J Dent Res. 1977:56:499-501.

30. Massaroto Barros B, Biasotto-Gonzalez DA, Bussadori SK, Gomes CAFP, Politti F. Is there a difference in the electromyographic activity of the masticatory muscles between individuals with temporomandibular disorder and healthy controls? A systematic review with meta-analysis. J Oral Rehabil. 2020:47:672-82.

31. Al-Saleh MA, Armijo-Olivo S, Flores-Mir C, Thie NM. Electromyography in diagnosing temporomandibular disorders. J Am Dent Assoc. 2012;143:351-62.

32. Szyszka-Sommerfeld L, Machoy M, Lipski M, Woźniak K. Electromyography as a means of assessing masticatory muscle activity in patients with pain-related temporomandibular disorders. Pain Res Manag. 2020;2020:9750915

33. Pietropaoli D, Ortu E, Giannoni M, Cattaneo R, Mummolo A, Monaco A. Alterations in surface electromyography are associated with subjective masticatory muscle pain. Pain Res Manag. 2019;2019:6256179.

\section{Publisher's Note}

Springer Nature remains neutral with regard to jurisdictional claims in published maps and institutional affiliations.
Ready to submit your research? Choose BMC and benefit from:

- fast, convenient online submission

- thorough peer review by experienced researchers in your field

- rapid publication on acceptance

- support for research data, including large and complex data types

- gold Open Access which fosters wider collaboration and increased citations

- maximum visibility for your research: over 100M website views per year

At BMC, research is always in progress.

Learn more biomedcentral.com/submissions 